PROCEEDINGS OF THE

AMERICAN MATHEMATICAL SOCIETY

Volume 79, Number 1, May 1980

\title{
THE SPECTRUM OF THE LAPLACIAN OF KÄHLER MANIFOLDS
}

\author{
BANG-YEN CHEN AND LIEVEN VANHECKE
}

ABSTRACr. We strengthen some results on the spectrum of the Laplacian for 0- and 1-forms [8], [9] on Kähler manifolds and give some new results for the 2-forms.

1. Introduction. Let $(M, J, g)$ be an $n$-dimensional Kähler manifold (all manifolds are assumed to be compact, connected and of complex dimension $n>1$ ) with complex structure $J$ and Kähler metric $g$. By $\Delta$ we denote the Laplacian acting on $p$-forms on $M$. Then we have the spectrum for each $p$,

$$
\operatorname{Spec}^{p}(M, g)=\left\{0 \geqslant \lambda_{1, p} \geqslant \lambda_{2, p} \geqslant \cdots \downarrow-\infty\right\},
$$

where each eigenvalue is repeated as many times as its multiplicity indicates. It is well known that $\operatorname{Spec}^{p}(M, g)=\operatorname{Spec}^{2 n-p}(M, g)$ and, immediate from Hodge theory, that $0 \in \operatorname{Spec}^{p}(M, g)$ if and only if the $p$ th Betti number $\beta_{p}(M) \neq 0$ (and 0 has multiplicity $\left.\beta_{p} \neq 0\right)$.

We denote by $\left(\mathbf{C} P^{n}, J_{0}, g_{0}\right)$ the $n$-dimensional complex projective space with the standard complex structure $J_{0}$ and the Fubini-Study metric $g_{0}$ of constant holomorphic sectional curvature $c$. One of the most interesting problems on spectrum is as follows: "Let $(M, J, g)$ be a Kähler manifold with $\operatorname{Spec}^{p}(M, g)=\operatorname{Spec}^{p}\left(C P^{n}, g_{0}\right)$ for a fixed $p$. Is it true that $(M, J, g)$ is holomorphically isometric to $\left(\mathbf{C} P^{n}, J_{0}, g_{0}\right)$ ?"

In [1], [5] it is proved that if $(M, J)$ is $\left(C P^{n}, J_{0}\right)$, then the answer to the problem is affirmative for $p=0$. In [8], [9] Tanno has proved that the answer is affirmative if $p=0$ and $n \leqslant 6$ or if $p=1$ and $8 \leqslant n \leqslant 51$.

The main purpose of this note is to prove the following.

THEOREM 1. Let $(M, J, g)$ be a Kähler manifold with $\operatorname{Spec}^{2}(M, g)=$ $\operatorname{Spec}^{2}\left(\mathbf{C} P^{n}, g_{0}\right)$. If $n \neq 8$, then $(M, J, g)$ is holomorphically isometric to $\left(\mathbf{C P}, J_{0}, g_{0}\right)$.

The method we use in the proof is different from the previous authors since the second cohomology group $H^{2}(M, \mathbf{R})$ and the generalized Chern classes $c_{1} \omega^{n-1}$ and $c_{1}^{2} \omega^{n-2}$ play very important roles in the proof.

2. Preliminaries. Let $M$ be a Kähler manifold of complex dimension $n$. If $\left(\theta^{1}, \ldots, \theta^{n}\right)$ form a local field of unitary coframes, the Kähler metric $g$ and the fundamental 2-form $\Phi$ are given respectively by $g=\frac{1}{2} \Sigma\left(\theta^{i} \otimes \bar{\theta}^{i}+\bar{\theta}^{i} \otimes \theta^{i}\right)$ and $\Phi=\frac{1}{2} \sqrt{-1} \sum \theta^{i} \wedge \bar{\theta}^{i}$. Let $\Omega_{j}^{i}=\sum R_{j k l}^{i} \theta^{k} \wedge \bar{\theta}^{l}$ be the curvature form of $M$. Then the curvature tensor $R$ is the tensor field with local components $R_{j k i}^{i}$. The Ricci tensor

Received by the editors December 5, 1978 and, in revised form, January 30, 1979.

AMS (MOS) subject classifications (1970). Primary 53C55; Secondary 58G99.

Key words and phrases. Spectrum, Laplacian, Kähler manifolds.

(c) 1980 American Mathematical Society 0002-9939/80/0000-02 16/\$02.25 
$S$ and the scalar curvature $\rho$ are given respectively by $S=\frac{1}{2} \Sigma\left(R_{i j} \theta^{i} \wedge \bar{\theta}^{j}+\bar{R}_{i j} \bar{\theta}^{i} \wedge\right.$ $\left.\theta^{j}\right)$ and $\rho=2 \sum R_{i i}$ where $R_{i j}=2 \sum R_{i k j}^{k}$. We denote by $\|R\|$ and $\|S\|$ the length of $R$ and $S$ respectively.

We define the $k$ th scalar curvature $\rho_{k}$ by

$$
\operatorname{det}\left(\delta_{i j}+t R_{i j}\right)=\sum_{k=0}^{n}\left(\begin{array}{l}
n \\
k
\end{array}\right) \rho_{k} t^{k}
$$

It is clear that $\rho_{0}=1, \rho_{1}=\rho / 2 n$,

$$
\rho_{2}=\frac{1}{4 n(n-1)}\left(\rho^{2}-2\|S\|^{2}\right), \ldots, \rho_{n}=\operatorname{det}\left(R_{i j}\right) .
$$

Let $\omega$ be the cohomology class represented by the fundamental form $\Phi$. Then $\omega$ is called the fundamental class and the last de Rham cohomology group $H^{2 n}(M, \mathbf{R})$ is generated by $\omega^{n}$. Let $c_{1}$ be the first Chern class of $M$. We put

$$
\omega^{n-k} c_{1}^{k}=\alpha_{k} \omega^{n}, \quad k=0,1, \ldots, n .
$$

Then, the $\alpha_{k}$ are real numbers. We state the following lemma for later use [3] (see [6] for the cohomologically Einstein case).

Lemma 1. Let $M$ be a Kähler manifold. Then we have

$$
\int_{M} \rho_{k} * 1=(2 \pi)^{k} \alpha_{k} \int_{M} * 1
$$

The Minakshisundaram-Pleijel-Gaffney formula for $\operatorname{Spec}^{p}(M, g)$ is given by

$$
\sum_{k=0}^{\infty} e^{\lambda x_{\rho} t} \underset{t \rightarrow 0}{\sim} \frac{1}{(4 \pi t)^{n}} \sum_{i=0}^{\infty} a_{i p} t^{i}
$$

where

$$
\begin{gathered}
a_{0, p}=\left(\begin{array}{c}
2 n \\
p
\end{array}\right) \int_{M} * 1, \\
a_{1, p}=\left\{\frac{1}{6}\left(\begin{array}{c}
2 n \\
p
\end{array}\right)-\left(\begin{array}{c}
2 n-2 \\
p-1
\end{array}\right)\right\} \int_{M} \rho * 1, \\
a_{2, p}=\int_{M}\left\{c_{1}(2 n, p) \rho^{2}+c_{2}(2 n, p)\|S\|^{2}+c_{3}(2 n, p)\|R\|^{2}\right\} * 1
\end{gathered}
$$

and

$$
\begin{gathered}
c_{1}(2 n, p)=\frac{1}{72}\left(\begin{array}{c}
2 n \\
p
\end{array}\right)-\frac{1}{6}\left(\begin{array}{c}
2 n-2 \\
p-1
\end{array}\right)+\frac{1}{2}\left(\begin{array}{c}
2 n-4 \\
p-2
\end{array}\right), \\
c_{2}(2 n, p)=-\frac{1}{180}\left(\begin{array}{c}
2 n \\
p
\end{array}\right)+\frac{1}{2}\left(\begin{array}{c}
2 n-2 \\
p-1
\end{array}\right)-2\left(\begin{array}{c}
2 n-4 \\
p-2
\end{array}\right), \\
c_{3}(2 n, p)=\frac{1}{180}\left(\begin{array}{c}
2 n \\
p
\end{array}\right)-\frac{1}{12}\left(\begin{array}{c}
2 n-2 \\
p-1
\end{array}\right)+\frac{1}{2}\left(\begin{array}{c}
2 n-4 \\
p-2
\end{array}\right) .
\end{gathered}
$$

The coefficients $a_{1, p}$ and $a_{2, p}$ are determined by Patodi [7]. 
3. Proof of Theorem 1. If $p=2$, the first three coefficients in the asymptotic expansion are given by

$$
\begin{aligned}
a_{0,2}= & n(2 n-1) \int_{M} * 1, \\
a_{1,2}= & \frac{2 n^{2}-13 n+12}{6} \int_{M} \rho * 1, \\
a_{2,2}= & \frac{1}{360} \int_{M}\left\{5\left(2 n^{2}-25 n+60\right) \rho^{2}-2\left(2 n^{2}-181 n+540\right)\|S\|^{2}\right. \\
& \left.+2\left(2 n^{2}-31 n+120\right)\|R\|^{2}\right\} * 1 .
\end{aligned}
$$

Now, assume that $\operatorname{Spec}^{2}(M, g)=\operatorname{Spec}^{2}\left(C P^{n}, g_{0}\right)$. Then we have

$$
\begin{gathered}
\operatorname{dim}_{\mathbf{C}} M=n, \quad \operatorname{dim} H^{2}(M, \mathbf{R})=\operatorname{dim} H^{2}\left(\mathbf{C} P^{n}, \mathbf{R}\right)=1, \\
\int_{M} * 1=\int_{\mathbf{C} P^{n}} * 1, \quad \int_{M} \rho * 1=\int_{\mathbf{C} P^{n}} \rho^{\prime} * 1, \\
a_{2,2}=a_{2,2}^{\prime},
\end{gathered}
$$

where $\rho^{\prime}, a_{2,2}^{\prime}, \ldots$ etc. are the corresponding quantities for $\left(\mathbf{C} P^{n}, J_{0}, g_{0}\right)$.

From Lemma 1, we have

$$
\begin{gathered}
\int_{M} \rho * 1=4 n \pi \alpha_{1} \int_{M} * 1 \\
\int_{M}\left(\rho^{2}-2\|S\|^{2}\right) * 1=16 n(n-1) \pi^{2} \alpha_{2} \int_{M} * 1 .
\end{gathered}
$$

On the other hand, since the second Betti number of $M$ is $1, c_{1}=a \omega$ for some real number $a$. Hence we have $\alpha_{2}=\alpha_{1}^{2}$. Consequently, (3.7) and (3.8) imply

$$
(n-1)\left(\int_{M} \rho * 1\right)^{2}=n\left(\int_{M} * 1\right) \int_{M}\left(\rho^{2}-2\|S\|^{2}\right) * 1 .
$$

Since $\left(\mathbf{C} P^{n}, J_{0}, g_{0}\right)$ has constant holomorphic sectional curvature $c$, we have

$$
\rho^{\prime}=n(n+1) c, \quad\left\|S^{\prime}\right\|^{2}=n(n+1)^{2} c^{2} / 2, \quad\left\|R^{\prime}\right\|^{2}=2 n(n+1) c^{2} .
$$

From (3.5), (3.7) and (3.10) we find $\alpha_{1}=(n+1) c / 4 \pi$. Using (3.3), (3.5) and (3.10) we have

$$
a_{2,2}^{\prime}=\frac{1}{360} n(n+1)\left(10 n^{4}-117 n^{3}+362 n^{2}-183 n-60\right) c^{2} \int_{M} * 1
$$

and so

$$
a_{2,2}^{\prime}=\frac{16 n \pi^{2}}{360(n+1)}\left(10 n^{4}-117 n^{3}+362 n^{2}-183 n-60\right) \alpha_{1}^{2} \int_{M} * 1
$$

Hence (3.7) implies

$$
a_{2,2}^{\prime}=\frac{1}{360 n(n+1)}\left(10 n^{4}-117 n^{3}+362 n^{2}-183 n-60\right) \frac{\left(\int_{M} \rho * 1\right)^{2}}{\int_{M} * 1}
$$


and using (3.9) we obtain

$$
a_{2,2}^{\prime}=\frac{1}{360\left(n^{2}-1\right)}\left(10 n^{4}-117 n^{3}+362 n^{2}-183 n-60\right) \int_{M}\left(\rho^{2}-\|S\|^{2}\right) * 1 .
$$

Then from (3.6) we find

$$
\begin{aligned}
& \int_{M}\left\{\left(n^{2}-1\right)\left(2 n^{2}-31 n+120\right)\right.\|R\|^{2} \\
&+4\left(2 n^{4}+16 n^{3}-44 n^{2}-91 n+120\right)\|S\|^{2} \\
&\left.-2\left(2 n^{3}+18 n^{2}-77 n+60\right) \rho^{2}\right\} * 1=0
\end{aligned}
$$

which is equivalent to

$$
\begin{aligned}
& \left(n^{2}-1\right)(n-8)(2 n-15) \int_{M}\left\{\|R\|^{2}-\frac{2}{n(n+1)} \rho^{2}\right\} * 1 \\
& \quad+4\left(2 n^{4}+16 n^{3}-44 n^{2}-91 n+120\right) \int_{M}\left\{\|S\|^{2}-\frac{\rho^{2}}{2 n}\right\} * 1=0 .
\end{aligned}
$$

Since $\|R\|^{2} \geqslant(2 / n(n+1)) \rho^{2}$ and $\|S\|^{2} \geqslant \rho^{2} / 2 n$, equation (3.12) gives $\|R\|^{2}=$ $(2 / n(n+1)) \rho^{2}$ and $\|S\|^{2}=\rho^{2} / 2 n$ if $n \neq 8$, from which we conclude that $(M, J, g)$ has constant holomorphic sectional curvature, say $\bar{c}$. From (3.5) it follows then that $c=\bar{c}$. Thus, $(M, J, g)$ is holomorphically isometric to $\left(\mathbf{C P}, J_{0}, g_{0}\right)$.

\section{Remarks.}

REMARK 1. A Kähler manifold $(M, J, g)$ is said to be cohomologically Einsteinian if $c_{1}=a \omega$ for some real number $a$. By a similar argument as in the proof of Theorem 1, we may prove the following.

THEOREM 2. If $(M, J, g)$ is a cohomological Einstein Kähler manifold and

$$
\operatorname{Spec}^{0}(M, g)=\operatorname{Spec}^{0}\left(C P^{n}, g_{0}\right) \text {, }
$$

then $(M, J, g)$ is holomorphically isometric to $\left(\mathrm{CP}^{n}, J_{0}, g_{0}\right)$.

THeOREM 3. If $(M, J, g)$ is a cohomological Einstein Kähler manifold and

$$
\operatorname{Spec}^{1}(M, g)=\operatorname{Spec}^{1}\left(C P^{n}, g_{0}\right), \quad n>7,
$$

then $(M, J, g)$ is holomorphically isometric to $\left(\mathrm{CP}^{n}, J_{0}, g_{0}\right)$.

RemarK 2. If $H^{2}(M, \mathbf{R})=\mathbf{R}$, then $M$ is automatically cohomologically Einsteinian.

COROLlaRY 4. If $(M, J, g)$ is a Kähler manifold with $H^{2}(M, \mathbf{R})=\mathbf{R}$ and $\operatorname{Spec}^{0}(M, g)=\operatorname{Spec}^{0}\left(C P^{n}, g_{0}\right) \quad\left(\operatorname{or}_{\operatorname{Spec}^{1}}(M, g)=\operatorname{Spec}^{1}\left(C P^{n}, g_{0}\right)\right.$ and $\left.n>7\right)$, then $(M, J, g)$ is holomorphically isometric to $\left(\mathrm{CP}^{n}, J_{0}, g_{0}\right)$.

REMARK 3. All complete intersections of dimension $n \geq 3$, all complete Kähler manifolds of positive curvature or of holomorphic pinching greater than $\frac{1}{2}[2]$ and all complete Kähler manifolds of positive holomorphic bisectional curvature [4] satisfy $H^{2}(M, \mathbf{R})=\mathbf{R}$. 
Remark 4. From the proofs of Theorems 2 and 3 we may also see that we may replace the condition of "cohomologically Einsteinian" by the weaker condition " $\alpha_{2}>\alpha_{1}^{2 "}$.

\section{REFERENCES}

1. M. Berger, P. Gauduchon and E. Mazet, Le spectre d'une variété riemannienne, Lecture Notes in Math., vol. 194, Springer-Verlag, Berlin and New York, 1971.

2. R. L. Bishop and S. I. Goldberg, On the topology of positively curved Kaehler manifolds. II, Tôhoku Math. J. 17 (1965), 310-318.

3. B. Y. Chen, Characterizations of Einstein Kaehler manifolds and applications, Atti Acad. Naz. Lincei Rend. Cl. Sci. Fis. Mat. Natur. 61 (1976), 592-595.

4. S. I. Goldberg and S. Kobayashi, Holomorphic bisectional curvature, J. Differential Geometry 1 (1967), 225-233.

5. A. Lascoux and M. Berger, Variétés Kählériennes compactes, Lecture Notes in Math., vol. 154, Springer-Verlag, Berlin and New York, 1970.

6. K. Ogiue, Generalized scalar curvatures of cohomological Einstein Kaehler manifolds, J. Differential Geometry 10 (1975), 201-205.

7. V. K. Patodi, Curvature and the fundamental solution of the heat operator, J. Indian Math. Soc. 34 (1970), 269-285.

8. S. Tanno, Eigenvalues of the Laplacian of Riemannian manifolds, Tôhoku Math. J. 25 (1973), 391-403.

9. ___ The spectrum of the Laplacian for 1-forms, Proc. Amer. Math. Soc. 45 (1974), 125-129.

Department of Mathematics, Michigan State University, East Lansing, Michigan 48824

Department of Mathematics, Katholiteke Universiteit leuven, Celestunenlan 200B, B-3030 LEUVEN, BeLGIUM 